\title{
Learning to Invert Local Binary Patterns
}

Felix Juefei-Xu

felixu@cmu.edu

Marios Savvides

msavvid@ri.cmu.edu
Carnegie Mellon University

Pittsburgh, Pennsylvania 15213

USA

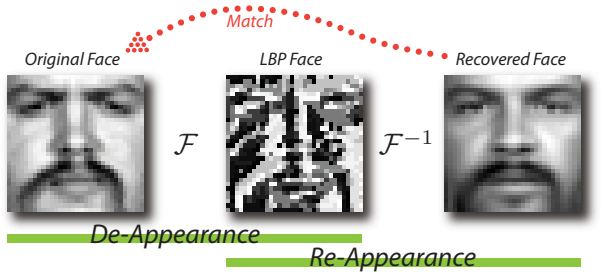

Figure 1: Flowchart of the method. The deappearance step uses LBP for the forward mapping $\mathcal{F}$ and obtains the LBP face (glyph) in the middle. The re-appearance step tries to learn the inverse mapping $\mathcal{F}^{-1}$ from the LBP domain to the pixel domain. The recovered face is of high fidelity as compared to the original face.

We have proposed to invert the local binary patterns (LBP) descriptor. The success of the inversion gives rise to two applications: face deappearance and re-appearance. The flowchart of the algorithm is shown in Figure 1.

The de-appearance, based on image-LBP forward mapping, is thorough in the sense that not only the identity information but also the soft-biometric information of the subject is removed. The intuition behind using LBP for deappearance is straightforward because LBP is a local difference operator and most of the COTS FRS cannot deal with LBP faces / glyphs. They either can not locate the LBP faces from the scene, or the match scores are horribly low.

The re-appearance yields face reconstruction with high fidelity and also enables secure application with a unique encryption key. The re-appearance is achieved by learning the inverse mapping of the LBP descriptors through an $\ell_{0^{-}}$ constrained coupled dictionary learning scheme that jointly learns two overcomplete dictionaries in both the pixel and the LBP domains such that inverse mapping from the LBP domain to the pixel domain is made possible without knowing the mapping function explicitly. The enforcement of the sparsity level as well as the sharing of sparse coefficient between the two domains are the added constraints that can uniquely determine the inverse mapping $\mathcal{F}^{-1}$.

Obtaining a consistent sparse encoding between the two domains allows for a more meaningful reconstruction. Given a novel deappearanced image $\mathbf{y}_{\mathrm{LBP}}$ in the LBP domain, we first obtain the sparse representation $\mathbf{x}$ in $\mathbf{D}_{\mathrm{LBP}}$. We then obtain the reconstruction using Dx. By forcing consistent sparse representations $\mathbf{x}$ during training, we optimize for a low reconstruction error for both domains jointly and simultaneously. A simple rearrangement can lead to solving the formulation using the standard KSVD dictionary learning approach [1] as previously observed [2]:

$$
\begin{array}{r}
\underset{\mathbf{D}, \mathbf{D}_{\mathrm{LBP}}, \mathbf{X}}{\arg \min }\left\|\left(\begin{array}{c}
\mathbf{Y} \\
\mathbf{Y}_{\mathrm{LBP}}
\end{array}\right)-\left(\begin{array}{c}
\mathbf{D} \\
\mathbf{D}_{\mathrm{LBP}}
\end{array}\right) \mathbf{X}\right\|_{F}^{2} \\
\text { subject to } \forall i,\left\|\mathbf{x}_{i}\right\|_{0} \leq K
\end{array}
$$

The procedure also comes naturally with high selectivity when reconstructing the faces with various LBP encryption keys. We have shown the effectiveness of our proposed approach on the FRGC ver 2.0 database which involves large-scale fidelity test and face verification experiments using the state-of-the-art commercial and academic face matchers.

[1] Michal Aharon, Michael Elad, and Alfred Bruckstein. K-SVD: An Algorithm for Designing Overcomplete Dictionaries for Sparse Representation. IEEE Transactions on signal processing, 54(11): 4311-4322, 2006.

[2] Zhuolin Jiang, Zhe Lin, and L.S. Davis. Label consistent K-SVD: Learning a discriminative dictionary for recognition. Pattern Analysis and $\mathrm{Ma}$ chine Intelligence, IEEE Transactions on, 35(11): 2651-2664, Nov 2013. 